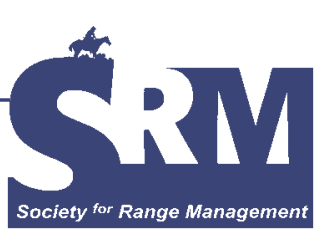

\title{
Wish Upon a Satellite: Applying GPS to Rangeland Management
}

By A. L. Hancock, E. K. Strand, and K. L. Launchbaugh

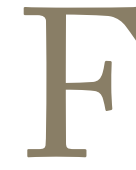

or years resource managers have used paper maps to document the location of important natural and human-imposed features. It is hard to find a desk, wall, or dashboard of a working range professional that is not adorned with some sort of map. Maps depict ownership boundaries, pasture fences, stream courses, and topographic features. The locations of monitoring plots, study sites, and range improvements are often stored on maps. Global Positioning Systems (GPS) now bring documenting spatial rangeland features to a new level of precision and accuracy. However, GPS systems come with a plethora of features and limitations that can cause confusion and frustration and make one long for the good old days of paper maps and compasses. In this manuscript we hope to dispel misconceptions and improve working knowledge of how GPS can be applied to rangeland management.

\section{Uses of GPS in Rangeland Management}

Range scientists adopted GPS technology shortly after it became available to aid in locating field plots, tracking wildlife and domestic livestock, and in recording known locations of invasive or rare species populations (Fig. 1). Rangeland managers and ranchers are now using GPS technology to accomplish many of their day-to-day tasks, including marking locations of range improvements, trend plots, green-line

This article has been peer reviewed.

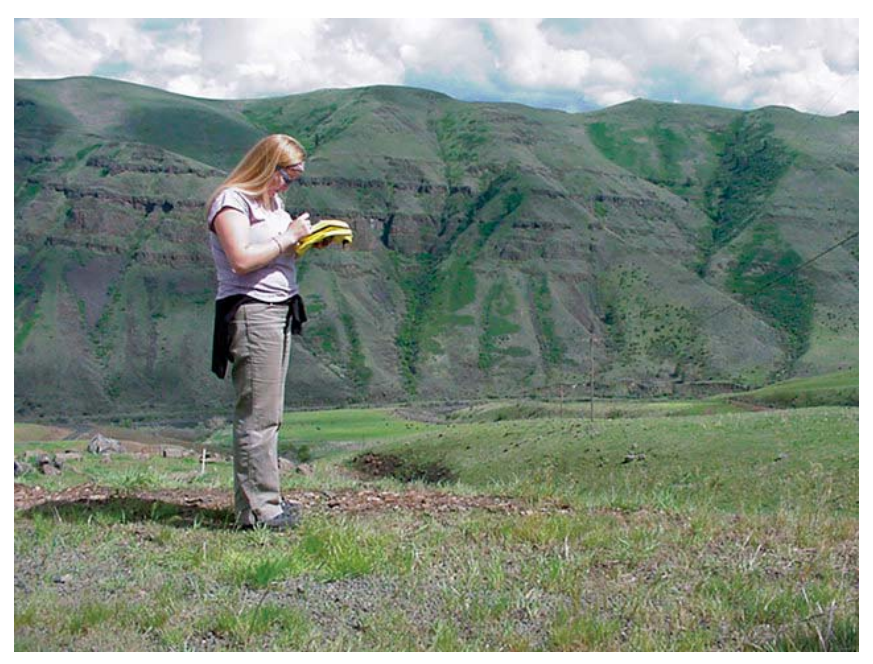

Figure 1. Today's rangeland specialists use GPS to document the location of many rangeland features including invasive plants, wildlife habitat, and range improvements.

surveys, and repeat photopoints. Historically, written directions on maps often marked monitoring points, with written directions or sketches on how to locate the plot. In most cases, legal coordinates (Township, Range, Section) were the only description of plot locations. The imprecise and sketchy locations led to thousands of hours spent simply trying to relocate monitoring plots. Even with a basic GPS unit, resource managers can now easily record or find a previous plot location within about 50 feet $(15 \mathrm{~m})$. 


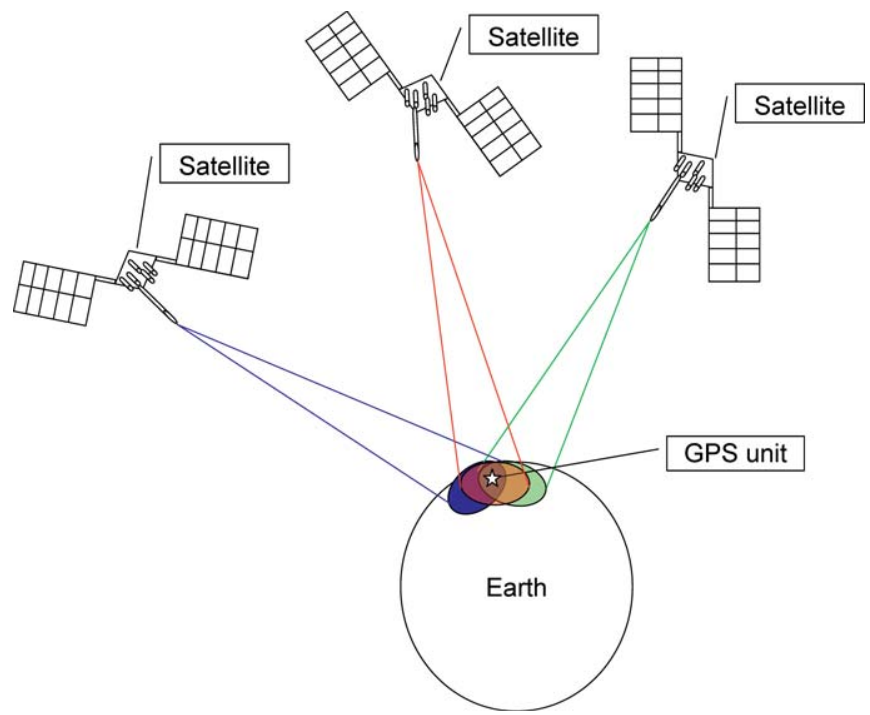

Figure 2. Global Positioning Systems (GPS) calculate their location based on a distance from at least 3 satellites through a process called "trilateration."

Locations and conditions of rangeland improvements, such as water tanks, fences, or creek crossings, can be collected and documented with GPS technologies and are an important part of the inventory of rangeland resource management tools. Some GPS units include the ability to add a description, or attribute, of specific locations such as the condition of a range improvement. Many invasive plants management programs include GPS tools to record the location of weeds, document management actions applied, and note the results achieved.

Information gained by GPS can also be linked to Geographic Information Systems (GIS) which are software programs designed to capture, display, analyze, retrieve, and store spatial information. Points and lines collected with a GPS unit can be displayed on maps created in a GIS. Sophisticated maps can also be created from layers such as slope, aspect, vegetation type, utilization level, and condition relative to streams and watering spots.

\section{What Is GPS and How Does It Work?}

The Global Positioning System (GPS) is a constellation of satellites orbiting the earth that constantly transmit signals of precise locations. The GPS in use today is called Navigation System with Timing and Ranging (NAVSTAR) and includes more than 24 solar-powered satellites. GPS units use a process called trilateration to accurately pinpoint locations on the earth's surface based on their distance from satellites of known location. Simply put, trilateration involves making measurements from intersecting circles of distance from satellites (Fig. 2). For example, if a GPS receiver was 11,000 miles from 1 satellite, there would be a finite number of places on the earth's surface (in the shape of a circle) where the location could be. If the same GPS receiver was 12,000 miles from a second satellite, the receiver could narrow down the location by the overlap from the 2 circles. The same would be true for a third and fourth satellite. In this manner, the more satellites a receiver can detect, the more accurate the position; GPS receivers require at least 3-4 satellites to $\log$ data points.

\section{How Accurate Is GPS?}

There are generally 3 grades of GPS units: recreational, mapping, and surveying. Most of the recreational grade units are marketed as being accurate from 20 to 50 feet (7-15 $\mathrm{m})$, but can incur higher inaccuracies. The mapping grade units are commonly accurate to 3 to 16 feet (1-5 m), and the survey grade units are usually accurate to less than 4 inches $(1-10 \mathrm{~cm})$, called "sub-foot" accuracy. Generally, the more accurate the unit is, the higher the cost. Most recreational grade units cost between $\$ 100-\$ 900$, whereas mapping grade units cost between $\$ 1,500-\$ 7,000$, and survey units generally cost upward to $\$ 45,000$. The accuracy of a location displayed on a GPS unit depends on several features related to the type and hardware of the unit itself, including the type of antenna, the number of channels the unit can receive, and the ability to differentially correct the location.

Antennas. A GPS unit receives signals from either an internal or an external antenna. Most of the recreational grade units employ internal antennas. Internal antennas, although not as accurate, are much more convenient. The mapping grade units generally come equipped with internal antennas, but have the option of an external antenna. The survey grade units generally have an external antenna. The advantage of an external antenna is that it allows a user to place the antenna where it can best acquire a satellite signal. For example, if a user was driving down a road, an external antenna could be placed on the outside of the vehicle for better reception.

Channels. The number of channels a unit has can affect a unit's accuracy. Most units of all grades currently have between 12-16 parallel channels. This means that these units can track 12-16 satellites simultaneously. Although a unit only needs 3 or 4 satellites to locate a position, the more satellites it can acquire, the more accurately it can narrow down its precise location.

Satellite Locations. The relative position of the satellites on the horizon affects the accuracy of the signal. If a satellite being tracked by a GPS unit is lower than $10^{\circ}-15^{\circ}$ on the horizon, it should be considered unusable. Because satellites are in a continuous orbit, and are constantly changing their location, acquiring a good satellite signal can be difficult or nearly impossible at certain times of the day. Some GPS units are capable of rejecting signals from satellites with poor position relative to their horizons. Mapping and survey grade units generally include software packages that calculate times on user specified dates when an adequate number of satellites will be available, which is useful in planning field activities that require GPS. One example is Trimble's "Pathfinder Office" (http://www.trimble.com/pathfinderoffice. shtml). These tools are useful in planning GPS acquisition to 
assure that 4 or more satellites will be available at the desired place and time of GPS data collection.

PDOP. "Position Dilution of Precision" is the expression of the effect that satellite positions has on GPS accuracy. It is difficult to acquire a precise location when satellites are clustered together in the GPS receiver's horizon because all the satellites are broadcasting similar location signals; thus, the circles of signal will overlap each other considerably. When the satellites are spread out over the sky, the precise location will be easier to acquire. In general, the lower the PDOP is, the more accurate the location. Commonly, a threshold PDOP of 6 is recommended, above which locations estimates are rejected because of potentially large inaccuracies. Mapping and survey grade units usually report PDOP, whereas recreational units usually do not.

WAAS and Other Differential Corrections. The process of applying corrections to data locations is called "differential correction" GPS or DGPS. Through DGPS the signal is corrected for atmospheric conditions, satellite orbit problems, and satellite clock drift. Most units of all grades allow for "real-time" differential correction, in which the current location displayed on a GPS receiver is adjusted based on the error of a simultaneous GPS reading at a base station of known location. One of the most common correction systems is the "Wide Area Augmentation System" (WAAS). Sometimes, however, a unit is too far away from a base station to receive a correction signal. When this happens, the data must be adjusted back in the office to obtain better accuracy. Most mapping and survey grade units allow for this "postprocessing," but recreational units generally do not. Base-station files for postprocessing can be accessed via the internet (http://www.fs.fed.us/database/gps/welcome.htm). The ability to postprocess data is one of the major price differences between recreational grade and mapping and survey grade units.

How do Environmental Conditions Affect GPS Accuracy?

The signal quality that reaches a GPS unit is affected by atmospheric and topographic conditions. Atmospheric gases, particularly water vapor, slow the speed of the signal, resulting in inaccurate estimations of position. Canyons, draws, or steep basins can inhibit the unit's ability to detect satellites that are lower on the horizon. Dense canopy covers in forests also distort the satellite signal and will cause some recreational grade units to produce errors over 165 feet $(50 \mathrm{~m})$. The errors in accuracy resulting from terrain and vegetation are called "multipath signals." That is, the satellite signal can be reflected off of inanimate objects such as trees, rocks, buildings, water bodies, the ground, etc. and then be picked up by a GPS unit, which results in significant distortion of the original signal. Some advanced mapping units and all survey grade units use a technology that is capable of filtering multipath signals and are more accurate under adverse conditions. Multipath filtering technology largely contributes to the accuracy and price differences between recreational grade units and mapping and survey grade units.

\section{What Kind of Display and Memory is Needed?}

Information on GPS units can be displayed either in color or grayscale. The recreational grade handheld units tend to come in grayscale, although most newer ones have optional color screens, but at an increased cost and reduced battery life. Most of the mapping and survey grade units come with full color screens that can easily be read in any light. The use of GPS units in rangeland settings generally requires screens that can be easily read in full sunlight. Because some GPS units can even display full topographic coverage of the area, screen readability is an important feature. Screen readability varies greatly from unit to unit, so make sure to try a unit in full sunlight before purchasing it.

The amount of memory on a GPS unit determines how much data, including maps, the unit can store. Many basic recreational units do not come with any capabilities to upload maps. These units have a predetermined amount of memory based on the number of waypoints and tracks they are programmed to handle. Intermediate and advanced recreational grade units include varying amount of memory for adding maps and storing data. Mapping and survey grade units have a virtually unlimited memory capacity because they can accept memory cards, so a user is limited only by the number of memory cards available. Units with more memory can upload and store more complicated data. For example, aerial photographs are commonly used with mapping quality units; however, photographs are extremely large files, too big for most recreational grade units. The memory capacity of a unit will affect its price but not the accuracy.

\section{Field GPS Settings and Data Management}

Data logging. Most units of all grades currently include data logging capabilities. This means that they are able to record positions, tracks, or routes. These data can then be stored in the unit or downloaded into a computer. An individual position recorded in the unit is called a waypoint, whereas a route is a collection of waypoints that define an intended path of travel. A track $\log$ is a collection of waypoints taken while the user is moving. Units vary considerably in the number of waypoints, routes, and track logs they can store. Most recreational grade units can store up to 500 waypoints, and between 20-50 routes. The survey and mapping grade units have a capacity that is only limited by their memory storage.

Data Dictionaries. Data dictionaries describe locations in a database and can be very useful in the field when a user wants to add descriptive information or attributes to object locations. For example, if a user is mapping locations of weed infestations, a data dictionary could include the date, species, phenology, infestation size, previous treatments, terrain, or the surrounding plant community. The information recorded in the data dictionary can be directly transferred from the GPS unit to a computer, avoiding the arduous task of entering field data 


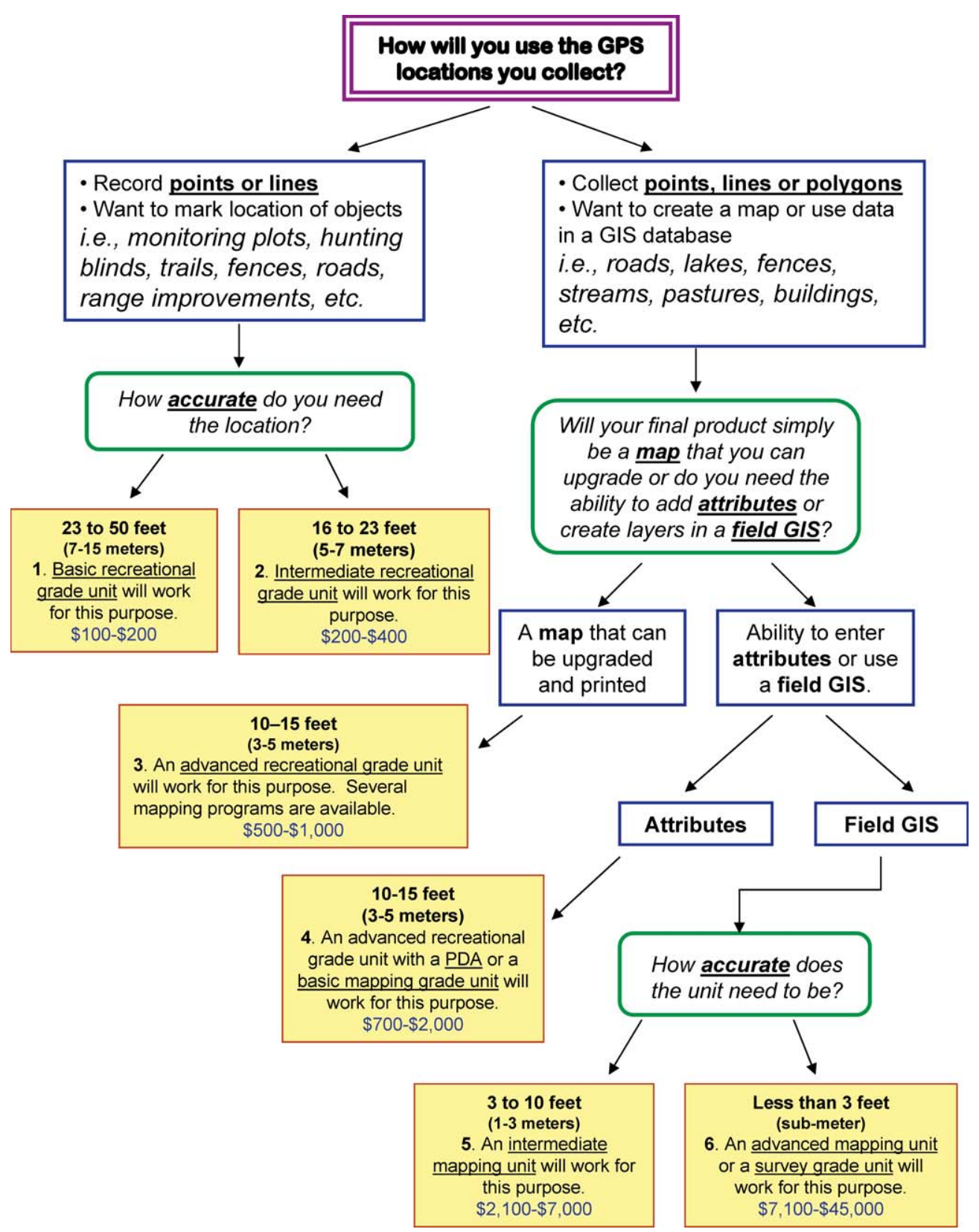

Figure 3. The selection of a GPS unit is based on accuracy needs, intended uses of data, and cost.

into the computer. Recreational grade GPS units generally do not have data dictionary capabilities unless they are connected to a palm computer or personal digital assistant (PDA).

Grids and Datums. GPS users must master the concept of grids and datums and understand how they apply to their fieldwork. A grid is a set of horizontal and vertical lines on a map that help determine absolute location on the earth; for example, latitude and longitude represent a grid system that builds a true relationship between map locations and those that correspond to on-the-ground points. There are several different grids or coordinate systems in use, and most GPS units can be set to report locations in a specified grid. The two most commonly used grids are Latitude and Longitude (lat/long) and Universal Transverse Mercator (UTM) pro- jection. Lat/long is a grid that is based on the measurement of angles from a reference line; the equator is the reference line for latitude and the Greenwich Meridian is the reference for longitude. UTM is a metric grid that divides the earth into 60 6-degree-wide zones. The advantage of using a metric grid such as UTM is that the location coordinates reported by the GPS unit directly relate to a distance on the ground. For example, if you move 100 meters to the north, the northing UTM coordinate has increased by 100 . On the other hand, most maps are displayed with a latitude/longitude grid, and setting the GPS unit to report latitude/longitude is convenient. Although it is possible to convert data between different grids, you must know the grid in which the location was originally collected. 
A datum is a geographic reference system of parameters and control points that accurately defines the three-dimensional shape of the earth. There are many different datums in use today, but the most common in North America are North American Datum 1927 (NAD 27), North American Datum 1983 (NAD 83), and World Geodetic System 1984 (WGS 84). Using the WGS 84 or NAD 83 datum generally results in slightly more accurate locations than older datums. Most of the older topographic maps produced by the United States Geological Survey (USGS) and the United States Forest Service (USFS) used NAD 27. It is important to know and correctly set the coordinate system and datum of the GPS unit. Not knowing the datum in which a point was collected can result in errors of up to a mile when trying to relocate a point using a different datum.

If you plan to incorporate GPS data in a Geographic Information System (GIS), it is advantageous to set the GPS unit to collect data in the same grid and datum that are used in the GIS. At minimum, it is important to note the datum in which you collected location data so that points can be reprojected and data can be shared.

\section{Relationship Between GPS and GIS}

GPS data can be used by itself - but many times it is integrated with a GIS program. GIS programs are capable of storing the spatial data collected by a GPS unit and displaying it as part of a map. The map can be tailored to an individual's preference, adding or removing layers of data as needed for a particular application. "Layers" represent a group of data that is categorized and displayed together. For example, a group of waypoints that make up a route, such as a road, might be displayed together in a layer. Another layer might be all the stock tanks on a ranch.

Field GIS. There are many different types of GIS programs and some have field-ready versions that can be incorporated into a GPS unit allowing data to be added and viewed in the field. Most mapping and survey grade units are able to handle a variety of field GIS programs. Some of the recreational grade units can be attached to a PDA to support such field GIS programs as ArcPad (http://www.esri.com). The use of a field GIS program reduces the need for significant data manipulation after returning from the field. In other words, the user can see exactly how the field data they are collecting fit with the other data used in a GIS.

Desktop GIS. GIS programs allow data from different sources to be combined. For example, data taken in the field with a GPS unit can be combined with topographic features, soils, and watershed characteristics. Many organizations such as federal land management agencies, states, and others host websites with spatial data available for download. For example, the Natural Resources Conservation Service has soil surveys available for download free (http://soildatamart.nrcs. usda.gov). A land manager could download a soil map of a parcel of land, use a GPS unit to record the location of fencelines, and then combine the two layers with a GIS program to determine the soil types within each pasture.
Aerial photographs are also commonly used with a GPS unit and incorporated into GIS data. There are many ways in which GPS data can be utilized within a GIS program, and it is important to recognize that although the two technologies can be used separate from one another, they can be used in combination to produce powerful analyses and maps.

\section{Which GPS Unit Is Best for Me?}

There are many different types and brands of GPS units available. Which ones work best for land managers? Which ones are most cost-effective? The answer to these questions depends on what needs to be accomplished with the unit. It also depends on the terrain and canopy cover in which the unit will be used and the user's accuracy needs. The decision tree in Figure 3 is designed to help rangeland managers decide what kind of unit may suit their needs best.

\section{Basic recreational unit}

Generally these units are meant for the occasional user that does not need highly accurate positions. They tend to be very rugged and user-friendly, but cannot download data from a website or upload data to a computer. Usually, these units have a long battery life and use batteries that can easily be replaced in the field. Most of the screens are either monochrome or grayscale, but tend to be fairly small. The units are lightweight, and can easily be carried in a backpack.

\section{Intermediate recreational unit}

These units have more features than the basic units, and take a little longer to learn how to use. Data collected with these units can be transferred to a computer and most will accept uploaded maps. Some of these units have color screens. The accuracy is generally better than the basic units, although they use the same types of technologies. These units are slightly larger than the basic units, but are rugged in construction. Most use $2 \mathrm{AA}$ batteries like the basic units, but the more expensive models can use rechargeable lithium ion (Li-ion) batteries that are expensive and can be difficult to obtain and/or replace.

\section{Advanced recreational unit}

These units generally have color screens and come loaded with several maps. They also have the ability to accept maps uploaded by the user. Like the intermediate units, some of the advanced units use $2 \mathrm{AA}$ batteries; others use a Li-ion battery. These units typically have other functions besides collection of location information; for example, they might have an electronic compass, barometric altimeter, or other features. These units typically either have a large memory
Side note: Some advanced recreational units can be attached to a personal digital assistant (PDA) or palm computer and are capable of adding attributes to features. They are also usually capable of handling a field version of a GIS program. 
capacity (> 100 megabytes) or are able to accept secure digital (SD) cards for data storage. These units have a shorter battery life due to the multiple functions and the color screen. The resolution of the screen (number of pixels) varies widely in this class of unit, but the screens tend to be larger than the previous two classes. Most of these units are rugged enough to be taken in the field, and the weights are generally comparable to the intermediate units.

\section{Basic mapping unit}

Basic mapping units are usually field computers or PDAs made more rugged for field use. They tend to be fairly accurate (comparable to the intermediate or advanced recreational unit), but lack some of the sophisticated technologies such as multipath signal rejection. Mapping units generally run a compact, field version of Microsoft Windows ${ }^{\circledR}$ and are capable of supporting field GIS programs. A user can create data dictionaries and add attributes to data points, with memory usually dictated only by the number and size of memory cards available to a user. These types of units are generally not as rugged or small as some of the recreational units and have large, full-color displays that reduce battery life. These units typically come with a rechargeable nickel metal hydride $(\mathrm{NiMH})$ or a Li-ion battery. Although the batteries are not meant to be changed in the field, most have the option of $12 \mathrm{~V}$ vehicle charging cables. The biggest difference between these types of units and the recreational units is the ability to process the data after it is collected, allowing for correction of inaccuracies incurred in field data collection.

\section{Intermediate mapping unit}

These units are very similar to the basic mapping units, but have a higher accuracy and are more field rugged. They are fully capable of running field GIS programs, and many have the ability to transfer data via a wireless network. These units are equipped with rechargeable batteries that last 5-8 hours.

\section{Advanced mapping unit/survey grade unit}

Even though there are significant differences between an advanced mapping unit and a survey grade unit, the authors group them here together for simplicity. These units have all the functions of the intermediate mapping units plus sophisticated technologies that allow for sub-foot accuracies. These units generally are not as rugged as some of the other units and weigh considerably more. They are also dependent on external antennae that require the use of cables and connectors. A user must be within a certain range of a base station for the technologies that allow for sub-foot accuracy to work. Cost can be prohibitive for these units.

\section{Summary}

The future is bright for the use of GPS in land management. As technology progresses, the cost of GPS units will undoubtedly come down and accuracy will increase. Currently, basic recreational grade units can be purchased for $\$ 100$ or less, making GPS technology very affordable. With a little experience, it would be easy to carry a GPS unit while out checking range resources, and record the location of important items such as poisonous plants, weed infestations, salt locations, broken fences, or wildlife sightings. The potential uses of GPS in land management are limited only by the user's imagination and the satellites in the sky!

Authors are Range and Natural Resource Specialist, Central Grasslands Research Extension Center, 4824 48th Ave SE, Streeter, ND 58483, amanda.hancock@ndsu.edu (Hancock), and Research Scientist (Strand) and Associate Professor (Launchbaugh), Department of Rangeland Ecology and Management, University of Idaho, ID 83844.

\section{For Further Reference}

Frair, J. L., S. E. Neilsen, E. H. Merrill, S. R. Lele, M. S. Boyce, R. H. M. Munro, G. B. Steinhouse, and H. L. BEYER. 2004. Removing GPS collar bias in habitat selection studies. Journal of Applied Ecology 41:201-212.

Gansкорт, D. 2001. Manipulating cattle distribution with salt and water in large arid-land pastures: a GPS/GIS assessment. $A p$ plied Animal Behavior Science 73:251-262.

Johnson, C. J., K. L. Parker, D. C. Heard, and M. P. GillINGHAM. 2002. Movement parameters of ungulates and scale specific responses. Journal of Animal Ecology 71:225-235.

Johnson, C. E., And C. C. BARton. 2004. Where in the world are my field plots? Using GPS effectively in environmental field studies. Frontiers in Ecology and Environment 2:475-482.

Witte, T. H., And A. M. Wilson. 2005. Accuracy of WAASenabled GPS for determination of position and speed over ground. Journal of Biomechanics 38:1717-1722. 